\title{
The theory of heating of the quantum ground state of trapped ions
}

\author{
Daniel F. V. James, \\ Theoretical Division T-4, Mail Stop B-268, \\ Los Alamos National Laboratory, Los Alamos, NM 87545, USA \\ July 7, 2018
}

\begin{abstract}
Using a displacement operator formalism, I analyse the depopulation of the vibrational ground state of trapped ions. Two heating times, one characterizing short time behaviour, the other long time behaviour are found. The short time behaviour is analyzed both for single and multiple ions, and a formula for the relative heating rates of different modes is derived. The possibility of correction of heating via the quantum Zeno effect, and the exploitation of the suppression of heating of higher modes to reduce errors in quantum computation is considered.
\end{abstract}

PACS numbers:42.50.Vk, 32.80.Pj, 03.67.Lx, 42.25.Kb LAUR 98-654

Individual or multiple ions can be confined in a radio-frequency Paul trap and using sophisticated laser techniques cooled to the quantum mechanical ground state [1], 2]. Such systems allow experimental preparation and measurement of non-classical motion states of the ions, and are therefore of great current interest in physics [3]. Furthermore important technological applications for such systems, such as the practical implementation of quantum computation, have recently attracted considerable attention [国, 5, 6, 目] . Quantum information can be stored in the internal quantum states of the ions (which constitute the quantum bits, or "qubits" of the computer), and, using ultra narrow bandwidth lasers, quantum gate operations can be realized between pairs of qubits using quantum states of the collective motion of the ions in the harmonic confining potential as a quantum information bus. If this bus were to become degraded by heating information would be lost, and so it is of great importance to maintain the ions in their motional ground state as long as possible. Of the many practical roadblocks standing in the way of success in realization of an ion trap quantum computer, one of the most important is the very fragile nature of this motional ground state, due to 
interactions with various ambient electromagnetic fields. In this letter I present a theoretical analysis of the depopulation of the motional ground state of ions due to such fields, which can be loosely characterized as heating (although it should be stressed that in this analysis relaxation to a thermal distribution is not considered).

Various analyses of decoherence mechanisms in ion trap quantum computers have been carried out [8, 9, 10, 11, 12]. Such effects as spontaneous emission from the internal states [9, 10, 11], or dephasing of the internal states due to the ions' zero-point motion [8], both of which effects can degrade or destroy quantum information, have been considered. Also the relaxation of an ion to a thermal state has been considered using a perturbation method, valid for describing long time behavior [13]; other work has been done on the effects of laser amplitude and phase stability [14, 15.

In this letter I adopt a rather different approach than that normally used in analysis of decoherence problems. When the electromagnetic fields causing the heating can be treated classically, the equations of motion reduce to a solvable system, namely a simple harmonic oscillator driven by a classical field. One can thus dispense with the density matrix/master equation formalism, using instead the interaction picture to solve exactly for the wavefunction describing the state of the ion. One can thus obtain an expression for the population remaining in the ground state as a function of time. An average of this population over an ensemble of realizations of the classical perturbing field (assumed to have Gaussian statistics) yields simple expressions for the time dependent behavior of the depopulation both for short and for long time limits.

In the interaction picture the Hamiltonian describing the interaction of a single ion of mass $M$ with a classical electric field $E(t)$ is given by the following formula

$$
\begin{aligned}
\hat{H} & =-e E(t) \hat{x} \\
& =i \hbar\left[u(t) \hat{a}^{\dagger}-u^{*}(t) \hat{a}\right]
\end{aligned}
$$

where $\hat{x}$ is the operator for the position of the ion and $u(t)=i e E(t) \exp \left(i \omega_{0} t\right) / \sqrt{2 M \hbar \omega_{0}}$, $e$ being the ion charge and $\hbar$ Planck's constant divided by $2 \pi$. I am considering only the motion of the ion along the weak confinement axis of an anisotropic trap, of the sort suitable for quantum computation; thus $E(t)$ represents the component of the electric field along that axis. The harmonic binding potential is characterized by the angular frequency $\omega_{0}$. The operators $\hat{a}\left(\hat{a}^{\dagger}\right)$ are the zero time annihilation (creation) operators for the harmonic motion of the ion in the harmonic well.

The dynamics of such a driven quantum harmonic oscillator can be solved exactly [16, 17]. The wave function at some instant $t$ is related to the initial wave function by the following expression

$$
|\psi(t)\rangle=\exp [i \phi(t)] \hat{D}[v(t)]|\psi(0)\rangle
$$

where $\hat{D}[v]=\exp \left[v \hat{a}^{\dagger}-v^{*} \hat{a}\right]$ is the displacement operator for coherent states of a harmonic oscillator, $\phi(t)$ is a phase factor (which turns out to be unimportant for the 
current problem) and the amplitude $v(t)$ is given by the formula

$$
\begin{aligned}
v(t) & \equiv \int_{0}^{t} u\left(t^{\prime}\right) d t^{\prime} \\
& =\frac{i e}{\sqrt{2 M \hbar \omega_{0}}} \int_{0}^{t} E\left(t^{\prime}\right) \exp \left(i \omega_{0} t^{\prime}\right) d t^{\prime} .
\end{aligned}
$$

As a figure of merit, let us introduce an average fidelity of the ground state, defined by the following formula:

$$
F(t)=\left\langle\left|\langle\psi(t) \mid \psi(0)\rangle_{q}\right|^{2}\right\rangle_{f}
$$

where $\langle\ldots\rangle_{q}$ denotes a quantum mechanical average, and $\langle\ldots\rangle_{f}$ an average over an ensemble of realizations of the classical random field $E(t)$. If one assumes that the ion is initially in the ground state, then its state evolves into a coherent state of amplitude $v(t)$. Thus the probability of remaining in the ground state can be found in closed form, and the fidelity of the ground state is given by the formula

$$
F(t)=\left\langle\exp \left[-|v(t)|^{2}\right]\right\rangle_{f}
$$

If one assumes Gaussian statistics for the classical random field $E(t)$ [18 (which can be justified by assuming that the field is due to many uncorrelated random sources, and then invoking the central limit theorem), the average over the field ensemble can be determined by performing an integration using the appropriate probability distribution. The result is as follows:

$$
\begin{aligned}
F(t) & =\left[1+2\left\langle|v(t)|^{2}\right\rangle_{f}\right. \\
& \left.+\left(\left\langle|v(t)|^{2}\right\rangle_{f}\right)^{2}-\left|\langle v(t) v(t)\rangle_{f}\right|^{2}\right]^{-1 / 2} .
\end{aligned}
$$

An alternative measure of the heating is the mean excitation number, defined by

$$
\bar{n}(t)=\left\langle\left[\left\langle\psi(t)\left|\hat{a}^{\dagger} \hat{a}\right| \psi(t)\right\rangle_{q}\right]\right\rangle_{f}
$$

Using eq.(22) and eq.(11.3-13) of ref. [17], this can be rewritten as

$$
\begin{aligned}
\bar{n} & =\left\langle\left[\left\langle\left. 0\left|\hat{n}+v^{*}(t) \hat{a}+\hat{a}^{\dagger} v(t)+\right| v(t)\right|^{2} \mid 0\right\rangle_{q}\right]\right\rangle_{f} \\
& =\left\langle|v(t)|^{2}\right\rangle_{f} .
\end{aligned}
$$

The correlation functions appearing in eqs.(6) and (8) can be evaluated using eq.(3). Using the symmetry property of the autocorrelation function, one obtains:

$$
\begin{aligned}
\left\langle|v(t)|^{2}\right\rangle_{f} & =\Omega^{2} t^{2} \int_{0}^{1}(1-x) \gamma_{E}(x t) \cos \left(x \omega_{0} t\right) d x \\
\left\langle v(t)^{2}\right\rangle_{f} & =\frac{\Omega^{2} t}{\omega_{0}} \exp \left(i \omega_{0} t\right) \\
& \times \int_{0}^{1} \gamma_{E}(x t) \sin \left[(1-x) \omega_{0} t\right] d x
\end{aligned}
$$


where $\gamma_{E}(\tau)=\langle E(t+\tau / 2) E(t-\tau / 2)\rangle_{f} /\left\langle E(t)^{2}\right\rangle_{f}$ is the degree of correlation of the field $E(t)$ (which is real) and the characteristic transition rate $\Omega$ is given by $\Omega=$ $\sqrt{e^{2}\left\langle E(t)^{2}\right\rangle_{f} / M \hbar \omega_{0}}$. Since I have implicitly assumed that $E(t)$ is stationary, $\left\langle E(t)^{2}\right\rangle_{f}$ is independent of time.

For an exponential degree of correlation given by $\gamma_{E}(\tau)=\exp (-|\tau| / T)$, the integrals given in eqs.(9) and (10) can be evaluated in closed form:

$$
\begin{aligned}
& \left\langle|v(t)|^{2}\right\rangle_{f}=\bar{n}(t)= \\
& \quad \frac{T}{\tau_{1}}\left[\exp (-t / T) \cos \left(\omega_{0} t+2 \phi\right)-\cos (2 \phi)+t / T\right], \\
& \left\langle v(t)^{2}\right\rangle_{f}= \\
& \quad \frac{T}{\tau_{1}} \exp \left(i \omega_{0} t\right)\left[\exp (-t / T) \sin (\phi)+\sin \left(\omega_{0} t-\phi\right)\right],
\end{aligned}
$$

where $\tan \phi=\omega_{0} T$ and the heating time $\tau_{1}$ is given by the formula

$$
\frac{1}{\tau_{1}}=\left(\frac{e^{2}\left\langle E(t)^{2}\right\rangle_{f}}{M \hbar \omega_{0}}\right) \frac{T}{1+\omega_{0}^{2} T^{2}} .
$$

Examples of the fidelity calculated using these results are shown in Fig.1. These show that revivals of the ground state populations can occur when the heating field has both a low amplitude and a long coherence time.

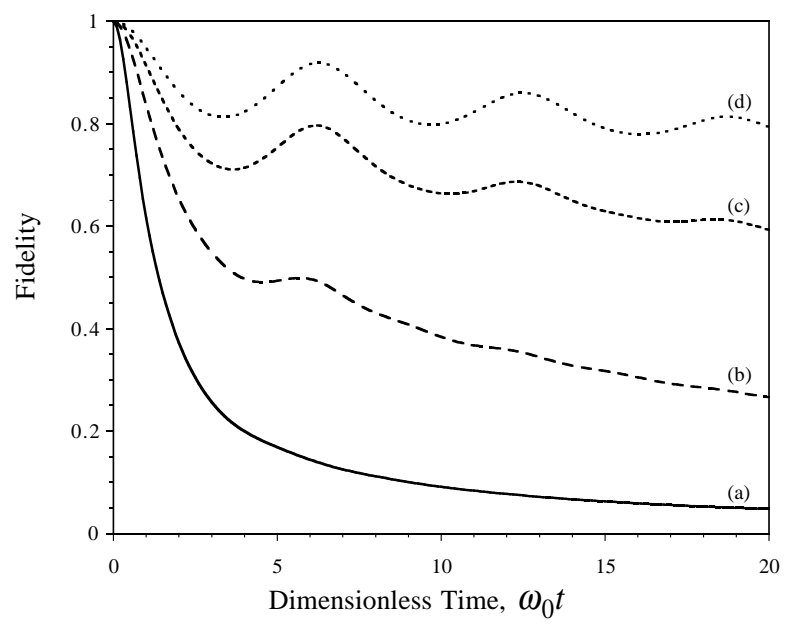

Figure 1: The fidelity of the ground state as a function of time, illustrating the results given in eqs.(6), (11) and (12). The parameters used were as follows: curve (a) $\omega_{0} T=1$, $\omega_{0} \tau_{1}=1$; curve (b) $\omega_{0} T=1, \omega_{0} \tau_{1}=8.5$; curve (c) $\omega_{0} T=1, \omega_{0} \tau_{1}=41$; curve (d) $\omega_{0} T=1$, $\omega_{0} \tau_{1}=128.5$. 
Two limiting cases are of interest, namely the behavior for short times and for very long times. For short times (i.e. $\left.t \ll T, 1 / \omega_{0}\right)$ the mean excitation number and fidelity are given by

$$
\bar{n}(t) \approx 1-F(t)=\left(\frac{1+\omega_{0}^{2} T^{2}}{2 T \tau_{1}}\right) t^{2}+O\left(t^{3}\right)
$$

This result, i.e. that for short times the "decay" of the ground state population is nonexponential, allows the possibility of maintaining the ion in its ground state via the quantum Zeno effect [19, 20]. If repeated measurements of the ground state population can be made on time scales much shorter than $1 / \omega_{0}$, then the ion will in principle remain in the ground state for a much longer time. However, as the quadratic behaviour persists for a short time only (see Fig.1), such a technique would be very difficult to implement. Furthermore, by invoking the time-energy uncertainty principle, one can see that measurements carried out on time scales much less than $1 / \omega_{0}$ will result in excitation of higher number states. Hence it is unlikely that the Zeno effect will be a practical method of error correction in these circumstances.

One can find asymptotic expressions for $F(t)$ and $\bar{n}(t)$ in the long time limit $t \gg$ $T, 1 / \omega_{0}$ :

$$
\begin{aligned}
& \bar{n}(t) \sim \frac{1}{\tau_{1}}\left(t-t_{0}\right)+O\left(\frac{1}{t^{3}}\right) \\
& F(t) \sim \frac{\tau_{1}}{t}-\frac{\tau_{1}^{2}\left(1-t_{0} / \tau_{1}\right)}{t^{2}}+O\left(\frac{1}{t^{3}}\right)
\end{aligned}
$$

where $t_{0}=T\left(1-\omega_{0}^{2} T^{2}\right) /\left(1+\omega_{0}^{2} T^{2}\right)$. These results are in qualitative agreement with that obtained earlier by Lamoreaux [13] using a perturbative density matrix approach.

In order to gain some insight into the order of magnitude of the heating effect, one can calculate a formula for the value of $\tau_{1}$ when the ambient electric field $E(t)$ is thermal, assuming a correlation time $T \approx \hbar / K_{B} \Theta \ll 1 / \omega_{0}$, (where $K_{B}$ is Boltzmann's constant and $\Theta$ is the temperature):

$$
\tau_{1} \approx \frac{3 c \epsilon_{0} \omega_{0} M K_{B}}{e^{2} \sigma \Theta^{3}}
$$

where $\sigma$ is the Stefan-Boltzmann constant and $\epsilon_{0}$ is the permittivity of free space. Using data from the mercury ion experiment described in ref. [1] (i.e. $M=3.29 \times 10^{-25}$ $\left.\mathrm{kg}, \omega_{0}=(2 \pi) 4.66 \mathrm{MHz}\right)$, a heating time $\tau_{1}=135 \mathrm{~ms}$ implies an effective temperature of $T=4.6 \mathrm{~K}$. This result should be treated with caution: the assumption that the random ambient field in the vicinity of the ion is thermal is not justified.

Let us now consider the case of multiple ions confined in a linear configuration. Because the ions are interacting via the Coulomb force, their motion will be strongly coupled. The small amplitude fluctuations are best described in terms of normal modes, each of which can be treated as an independent harmonic oscillator [7]. If there are $N$ ions in the trap, there will be a total of $N$ such modes. I will number these modes 
in order of increasing resonance frequency, the lowest $(p=1)$ mode being the center of mass mode, in which the ions oscillate as if rigidly clamped together. In the quantum mechanical description, each mode is characterized by creation and annihilation operators $\hat{a}_{p}^{\dagger}$ and $\hat{a}_{p}$ (where $\left.p=1, \ldots N\right)$. The Hamiltonian in this case is given by the expression

$$
\begin{aligned}
\hat{H} & =-e \sum_{n=1}^{N} E_{n}(t) \hat{x}_{n}(t) \\
& =i \hbar \sum_{p=1}^{N}\left[u_{p}(t) \hat{a}_{p}^{\dagger}-u_{p}^{*}(t) \hat{a}_{p}\right]
\end{aligned}
$$

where $E_{n}(t)$ is the electromagnetic field at the $n$-th ion of the string of ions, $\hat{x}_{n}(t)$ is the operator for the displacement of the $n-t h$ ion from its equilibrium position and

$$
u_{p}(t)=\frac{i e}{\sqrt{2 M \hbar \omega_{0} \sqrt{\mu_{p}}}} \sum_{n=1}^{N} E_{n}(t) b_{n}^{(p)} \exp \left(i \sqrt{\mu_{p}} \omega_{0} t\right)
$$

In eq.(19), $b_{n}^{(p)}$ is the $n$-th element of the $p$-th normalized eigenvector of the ion coupling matrix [7], $\mu_{p}$ being its eigenvalue. Again the evolution of the state of the ions can be solved exactly:

$$
|\psi(t)\rangle=\exp [i \Phi(t)] \prod_{p=1}^{N} \hat{D}_{p}\left[v_{p}(t)\right]|\psi(0)\rangle,
$$

where $\hat{D}_{p}\left[v_{p}\right]=\exp \left[v_{p} \hat{a}_{p}^{\dagger}-v_{p}^{*} \hat{a}_{p}\right]$ is the displacement operator for the $p$-th mode, and

$$
v_{p}(t)=\int_{0}^{t} u_{p}\left(t^{\prime}\right) d t^{\prime}
$$

As before, one can find a closed form expression for the fidelity of the ground state of the string of ions. For multiple ions, the mean excitation number is given by a formula analogous to eq.(16), where the characteristic decay time is given by:

$$
\tau_{N}=\tau_{1}\left[\sum_{p=1}^{N} \sum_{m, n=1}^{N} \frac{b_{m}^{(p)} b_{n}^{(p)}}{\sqrt{\mu_{p}}} \gamma_{m n}\right]^{-1}
$$

$\gamma_{m n}$ being the degree of coherence of the field at the positions of ions $n$ and $m$ evaluated for zero time delay [17] (the field having been assumed to be cross-spectrally pure [21, 22]), and the coherence time $T$ has been assumed to be much less than $1 / \omega_{0}$. In the coherent limit $\left(\gamma_{m n}=1\right)$, the formula for $\tau_{N}$ reduces to the following simple expression

$$
\tau_{N}=\frac{\tau_{1}}{N}
$$


while in the incoherent limit $\left(\gamma_{m n}=\delta_{m, n}\right), \tau_{N}$ is given by the formula

$$
\tau_{N}=\tau_{1}\left[\sum_{p=1}^{N} \frac{1}{\sqrt{\mu_{p}}}\right]^{-1} .
$$

The sum can be worked out from the eigenvalues $\mu_{p}$, which must be determined numerically in general. The results are shown in fig.2. The separation of ions is generally of the order of a few tens of microns. The coherence length of thermal light is $\ell_{\text {coh }} \approx \hbar c / K_{B} \Theta=2.3 \mathrm{~mm} / \Theta$; at low temperatures it is much longer than the ion separation. Therefore it is the coherent limit that is the important one, at least for small numbers of ions.

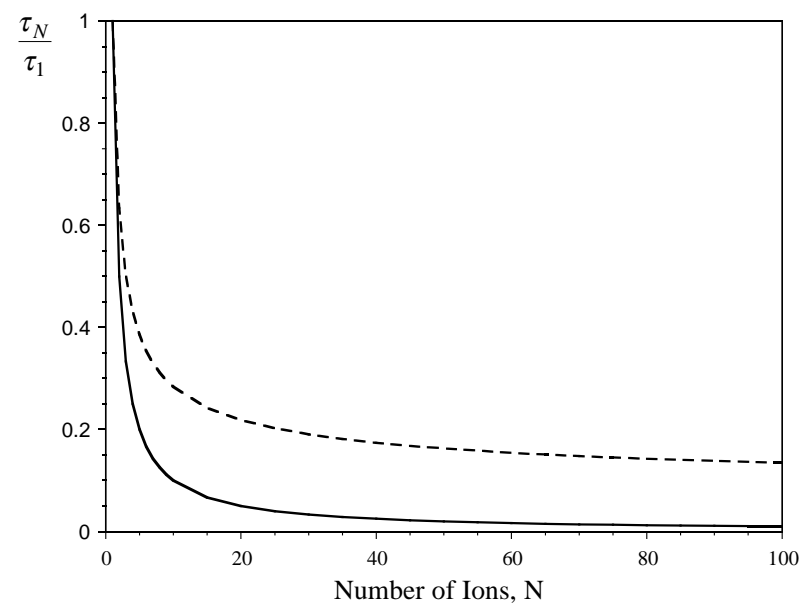

Figure 2: The heating time of the ground state for different numbers of ions, in the spatially coherent (plain curve) and spatially incoherent (dashed curve) limits for the ambient electric field.

One can also consider the mean excitation numbers of the different modes. These quantities are given by expressions analogous to eq.(8), with the heating time of the $p$-th mode, when there are $N$ ions in the string, given by

$$
\tau_{N, p}=\frac{\sqrt{\mu_{p}}}{\sum_{m, n=1}^{N} b_{m}^{(p)} b_{n}^{(p)} \gamma_{m n}} \tau_{1} .
$$

In the coherent and incoherent limits, the heating times of the different modes are given

$$
\begin{aligned}
\tau_{N, p} & =\left\{\begin{array}{ll}
\tau_{1} / N & p=1 \\
\infty & p>1 .
\end{array}\right. \text {. coherent } \\
\tau_{N, p} & =\sqrt{\mu_{p}} \tau_{1} \text { incoherent. }
\end{aligned}
$$


The heating times for the coherent case is potentially a very important result. Only the lowest $(p=1)$ centre of mass mode will be heated up by spatially coherent fields. Thus the state of the ion oscillations is given by:

$$
|\psi(t)\rangle=\exp i \phi(t)\left\{\left|v_{1}(t)\right\rangle_{1} \otimes|0\rangle_{2} \otimes|0\rangle_{3} \otimes \ldots \otimes|0\rangle_{N}\right\}
$$

the modes other than the center of mass mode remaining in their ground states. It should therefore be possible to perform quantum logic operations without degradation due to heating, simply by utilizing these higher modes as the quantum information bus rather than the center of mass mode [23].

The author would like to thank Albert Petschek for reading various versions of the manuscript and contributing many useful comments, and Mark Gulley, Richard Hughes, Steve Lamoreaux, Sara Schneider, Dave Wineland and Andrew White for other useful conversations and correspondence. This work was funded by the National Security Agency.

\section{References}

[1] F. Diedrich, J.C. Bergquist, W.M. Itano, and D.J. Wineland, Phys. Rev. Lett. 62, 403 (1989).

[2] C. Monroe et al., Phys. Rev. Lett. 75, 4011 (1995).

[3] D. Leibfried et al., J. Mod. Opt. 44, 2485 (1997).

[4] J.I. Cirac and P. Zoller, Phys. Rev. Lett. 74, 4094 (1995).

[5] C. Monroe et al., Phys. Rev. Lett. 75, 4714 (1995).

[6] A.M. Steane, Appl. Phys. B 64, 623 (1997).

[7] D.F.V. James, Appl. Phys. B 66, 181 (1998).

[8] A. Garg, Phys. Rev. Lett. 77, 964 (1996).

[9] R.J. Hughes et al., Phys. Rev. Lett. 77, 3240 (1996).

[10] M.B. Plenio and P.L. Knight, Proc. Roy. Soc. Lond. A 453, 2017 (1997).

[11] D.F.V. James et al., in Photonic Quantum Computing, Proceedings of SPIE 3076, edited by S.P. Hotaling and A.R. Pirich (SPIE, Bellingham, 1997), pp. 42-50.

[12] D.J. Wineland et al., Technical report, National Institute of Standards and Technology, Boulder, (unpublished), submitted to Rev. Mod. Phys.

[13] S.K. Lamoreaux, Phys. Rev. A 56, 4970 (1997). 
[14] S. Schneider and G. J. Milburn, "Decoherence in ion traps due to laser intensity and phase fluctuations", Phys. Rev. A, in the press, (1998); quant-ph/9710044.

[15] M. Murao and P. L. Knight, quant-ph/9803041.

[16] R.J. Glauber, Phys. Rev. 131, 2766 (1963).

[17] L. Mandel and E. Wolf, Optical Coherence and Quantum Optics (Cambridge University Press, Cambridge, 1995).

[18] J.W. Goodman, Statistical Optics (John Wiley and Sons, New York, 1985).

[19] W.H. Zurek, Phys. Rev. Lett. 53, 391 (1984).

[20] W.M. Itano, D.J. Heinzen, J.J. Bollinger, and D.J. Wineland, Phys. Rev. A 41, 2295 (1990).

[21] L. Mandel, J. Opt. Soc. Amer. 51, 1342 (1961).

[22] D. F. V. James and E. Wolf, Opt. Commun. 138257 (1997).

[23] This result was derived following correspondence with D. J. Wineland, who has independently reached similar conclusions (see: B. E. King et al., "Initializing the collective motion of trapped ions for quantum logic", submitted to Phys. Rev. Lett. (1998). 